\title{
Angle-resolved x-ray circular and magnetic circular dichroisms: Definitions and applications
}

\author{
S. Y. Tong \\ Department of Physics, The University of Hong Kong, Hong Kong \\ X. Guo \\ Department of Physics and Laboratory for Surface Studies, University of Wisconsin-Milwaukee, Milwaukee, Wisconsin 53201 \\ J. G. Tobin \\ Chemistry and Materials Science Department, Lawrence Livermore National Laboratory, Livermore, California 94550 \\ G. D. Waddill \\ Department of Physics, University of Missouri-Rolla, Rolla, Missouri 65401
}

(Received 12 March 1996)

\begin{abstract}
We introduce definitions of angle-resolved x-ray circular dichroism (ARXCD) and magnetic x-ray circular dichroism (ARMXCD). As defined, the much larger effect of circular dichroism (ARXCD) is separated from the smaller magnetic (ARMXCD) effect. In all materials, ARXCD is zero along mirror planes while nonzero elsewhere. ARMXCD is nonzero only in magnetic materials. The measurement and analysis of ARMXCD allow element specific surface magnetism and surface structure as well as their inter-relationship to be studied as functions of the outgoing electron's direction. [S0163-1829(96)05642-1]
\end{abstract}

\section{INTRODUCTION}

With the availability of tunable, circularly polarized $\mathrm{X}$-rays from synchrotron radiation sources and the promise of even greater brightness from new generation rings, magnetic x-ray circular dichroism (MXCD) has gained prominence as a tool to study element specific magnetic properties of thin films, interfaces, and surfaces. ${ }^{1-11}$ The majority of the experiments performed to date are based on absorption: An asymmetry factor, defined as $\left(\sigma_{\text {parallel }}-\sigma_{\text {antiparallel }}\right) /$ $\left(\sigma_{\text {parallel }}+\sigma_{\text {antiparallel }}\right)$, is measured as a function of photon energy. Here, $\sigma_{\text {parallel }}$ or $\sigma_{\text {antiparallel }}$ is an absorption coefficient which depends on whether the x-ray helicity is parallel or antiparallel to the direction of magnetization. Implicit in this definition is the assumption that the absorption coefficient depends on the relative and not the individual directions of helicity and magnetization. This assumption fails in asymmetry experiments based on angle-resolved photoelectron emission.

Recently, experiments and dynamical calculations were carried out to study the magnetization-helicity induced asymmetry in angle-resolved photoelectron emission. ${ }^{7-10}$ Analogous to nonmagnetic angle-resolved photoelectron diffraction, ${ }^{12}$ the magnetic counterpart measures the magnetization-helicity induced asymmetry either as a function of photon energy at constant outgoing electron direction or as a function of outgoing electron direction at constant photon energy. The incident photon direction is either held fixed or it varies in these measurements. Again, an important configuration is that the wave vector of the $\mathrm{x}$ ray is either parallel or antiparallel to the magnetization. In this paper, only these two configurations are considered. The measurements actually record two effects: (1) angle-resolved x-ray circular dichroism (ARXCD), which is present in all materials, and (2) angle-resolved x-ray magnetic circular dichroism
(ARMXCD), which is present only in magnetic materials. ${ }^{13-18}$ Since ARXCD (or known as circular dichroism angular distribution CDAD) is usually much larger than ARMXCD (also known as MCDAD), to study magnetic properties, it is necessary to distinguish and separate out the two effects. In this paper, we introduce definitions of ARMXCD and ARXCD having the following properties: (1) ARMXCD is zero everywhere for a nonmagnetic material, (2) ARXCD is nonzero except along mirror planes of a system (system $=$ crystal + incident photon direction). Along mirror planes, ARXCD is zero in all materials. (3) The integral of ARMXCD over all directions recovers the definition of MXCD used in absorption experiments. The integral of ARXCD over all directions is zero in chirally invariant systems. (4) ARMXCD and ARXCD depend on the individual directions of helicity and magnetization-not just their relative directions-except along mirror planes of the system.

\section{FINAL-STATE SPIN-RESOLVED INTENSITIES}

Consider, as an example, a circularly polarized light incident on a crystal with its propagation vector $\hat{k}$ lying in a symmetry plane of the crystal. We define the light's helicity with respect to its propagation vector $\hat{k}$ : for positive $(P)$ helicity, an observer facing the oncoming wave sees a counterclockwise rotation of the electric-field vectors. For a negative $(N)$ helicity, an observer facing the oncoming wave sees a clockwise rotation of the electric-field vectors. The magnetization and electron spin are defined as $(+)$ and $(\uparrow)$ if they are parallel to $\hat{k}$ of a positive $(P)$ helicity light. The magnetization and electron spin are $(-)$ and $(\downarrow)$ if they are antiparallel to $\hat{k}$ of a positive $(P)$ helicity light. In a ferromagnetic material, the minority electrons have $(\uparrow)$ spin if the magnetization is $(+)$ and $(\downarrow)$ if the magnetization is $(-)$. In general, there are eight inequivalent intensities at a given outgoing 


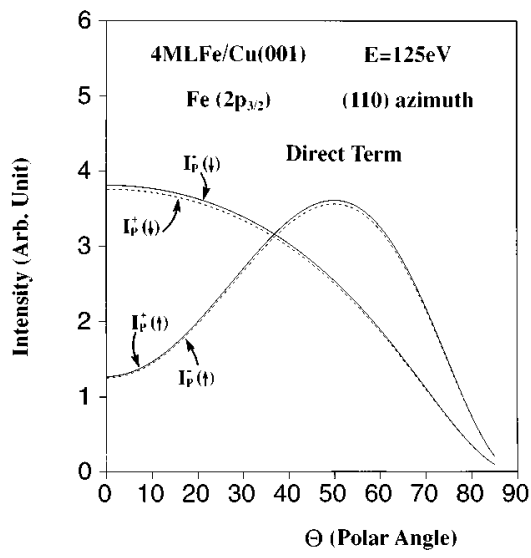

FIG. 1. Photoemission cross-section calculations for the $\mathrm{Fe}$ $\left(2 p_{3 / 2}\right)$ state showing spin-resolved intensities as a function of polar angles along the (110) mirror plane for the direct term, i.e., no final-state scattering. Polar angle $\theta=0^{\circ}$ is normal to the surface.

electron direction $\hat{\Omega}$ in a spin- and angle-resolved experiment. These are $I_{P}^{+}(\hat{\Omega})(\uparrow, \downarrow), I_{P}^{-}(\hat{\Omega})(\uparrow, \downarrow), I_{N}^{+}(\hat{\Omega})(\uparrow, \downarrow)$, and $I_{N}^{-}(\hat{\Omega})(\uparrow, \downarrow)$. However, along a mirror plane of the system, the eight quantities reduce to four because of the equalities:

$$
\begin{aligned}
& I_{P}^{+}(\hat{\Omega})(\uparrow)=I_{N}^{-}(\hat{\Omega})(\downarrow), \\
& I_{P}^{+}(\hat{\Omega})(\downarrow)=I_{N}^{-}(\hat{\Omega})(\uparrow), \\
& I_{P}^{-}(\hat{\Omega})(\uparrow)=I_{N}^{+}(\hat{\Omega})(\downarrow), \\
& I_{P}^{-}(\hat{\Omega})(\downarrow)=I_{N}^{+}(\hat{\Omega})(\uparrow) .
\end{aligned}
$$

We illustrate the angular dependence of these quantities by results of a spin-dependent photoelectron diffraction calculation on a thick $\mathrm{Cu}(001)$ slab with four ferromagnetic Fe layers on top. The Fe layers are assumed to be pseudomorphic with the $\mathrm{Cu}$ layers. The surface spacings between the $\mathrm{Fe}$ layers are given in Ref. 7 where comparisons with experiment are reported. For this specific example, we choose the light incident normal to the surface and the magnetization in the Fe film is assumed to be perpendicular to the surface. However, the definitions and equations introduced in this paper are valid for arbitrary directions of the incident light (with the magnetization direction pointing either parallel or antiparallel to this direction). For light at other angles of incidence, the mirror planes of the system will change according to the new geometry. Details of the spin-dependent photoelectron diffraction calculation using a relativistic Hamiltonian are given in Appendix A.

In Fig. 1, we show the four quantities on the left side of Eqs. (1)-(4), calculated for electron directions along the (110) mirror plane. The intensities are for emission from the $\mathrm{Fe}\left(2 p_{3 / 2}\right)$ initial state, at a final-state electron energy of 125 $\mathrm{eV}$. The intensities are plotted as a function of the outgoing electron's polar angle. In Fig. 1, the direct terms, i.e., no final-state scattering, are shown. The direct terms are related to the differential photoemission cross sections of an isolated Fe atom, except that a correction factor must be applied. This correction factor is due to the difference between the perpendicular component of the wave vector in a solid (complex

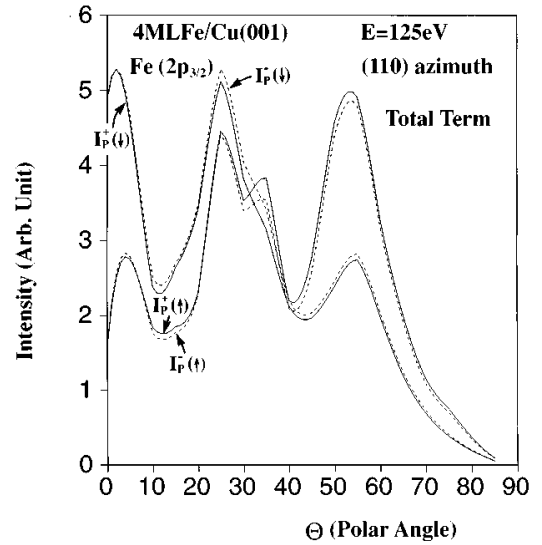

FIG. 2. Same as Fig. 1, except the spin-resolved total term intensities are shown. Note the additional diffraction features in the total term intensities.

due to an optical potential) and in vacuum (real). This correction factor also depends on the direction of the photoelectron since an outgoing wave is increasingly damped inside a solid with larger angles from the surface normal (e.g., see Sec. IV of Ref. 19). In terms of spin polarization, Fig. 1 shows that at the normal direction, $I_{P}^{+}(\uparrow)$ is 3.04 times smaller than $I_{P}^{+}(\downarrow)$. The two intensities cross at $\theta=37^{\circ}$, after that $I_{P}^{+}(\uparrow)$ is larger. Both intensities taper off at glancing emission angles because of the surface boundary condition (see Ref. 19).

Figure 2 shows the same four quantities with final-state multiple scatterings included. Comparing Figs. 1 and 2, we see that final-state diffraction effects are about $10-30 \%$, but can be as large as $100 \%$ at angles where the direct term is small. We have also calculated similar curves for the $\mathrm{Fe}\left(2 p_{1 / 2}\right)$ initial state. Again, both the direct and total (with final-state diffraction) intensities show a strong spinpolarization dependence on the outgoing direction.

Off-mirror plane directions, Eqs. (1)-(4) no longer hold due to the large angle-resolved circular dichroism effects. For example, ARXCD causes the intensity of $(\uparrow)$ spin and $(P)$ helicity to be different from that of $(\downarrow)$ spin and $(N)$ helicity. In Figs. 3(a) and 3(b), we show the polar angular dependence of the eight quantities in Eqs. (1)-(4) along a nonmirror plane azimuth: $20^{\circ}$ from (110) towards (010). From this figure, we see that while reversing the magnetization direction produces a 1-2\% effect, changing the helicity produces a $20-40 \%$ effect. In the next section, we shall provide asymmetry definitions which separate out these two effects.

\section{DEFINING ANGLE-RESOLVED XCD AND MXCD}

Almost all experiments using circularly polarized light carried out so far measure summed-spin intensities. These are obtained by adding the corresponding $(\uparrow)$ and $(\downarrow)$ spin terms in Eqs. (1)-(4). Along a nonmirror plane direction, there are four inequivalent summed-spin quantities: $I_{P}^{+}(\hat{\Omega})$, $I_{P}^{-}(\hat{\Omega}), I_{N}^{+}(\hat{\Omega})$, and $I_{N}^{-}(\hat{\Omega})$. It is necessary to note that although in both $I_{P}^{+}(\hat{\Omega})$ and $I_{N}^{-}(\hat{\Omega})$, the helicity and magnetization are parallel, these quantities are different. Figure 4 shows these four quantities along a nonmirror plane azimuth: 

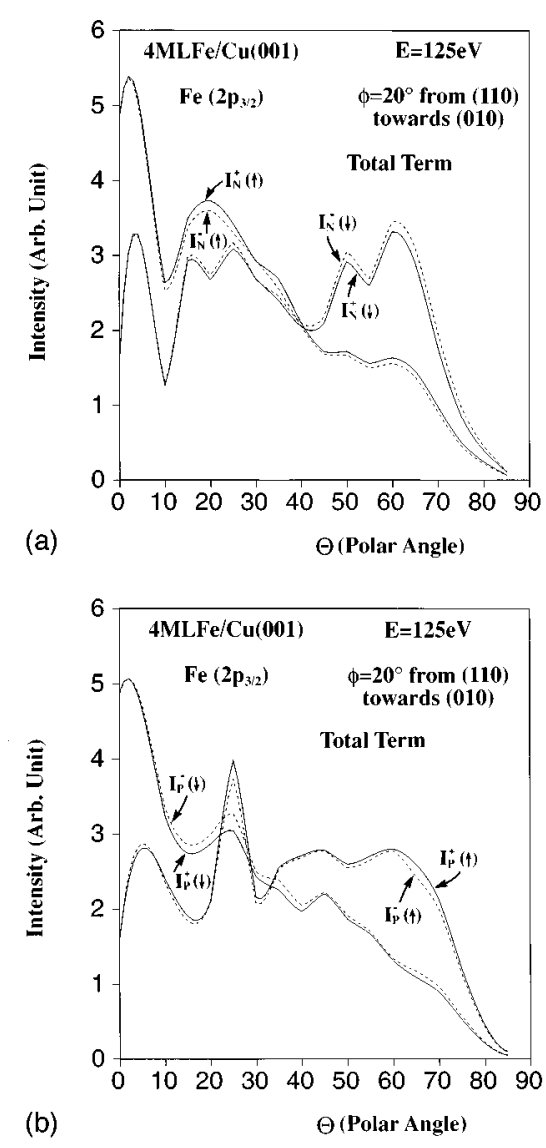

FIG. 3. Same as Fig. 2, except the spin-resolved total term intensities are shown along a nonmirror plane azimuth. There are eight spin-resolved quantities, with (a) four with negative helicity and (b) four with positive helicity.

$\varphi=20^{\circ}$ from (110). We note that the difference between the "parallel" terms $I_{P}^{+}(\hat{\Omega})$ and $I_{N}^{-}(\hat{\Omega})$ contains both angleresolved XCD and MXCD effects. The same is true for the difference between the two "antiparallel" terms $I_{N}^{+}(\hat{\Omega})$ and $I_{P}^{-}(\hat{\Omega})$. On the other hand, the "parallel" term $I_{P}^{+}(\hat{\Omega})$ and the "antiparallel" term $I_{P}^{-}(\hat{\Omega})$ differ only by the angle-

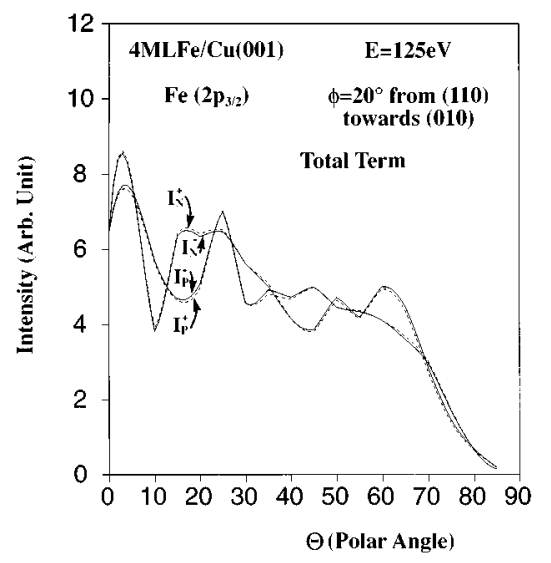

FIG. 4. Same as Fig. 3, except the spin-summed total term intensities are shown along a nonmirror plane azimuth. The eight spin-resolved quantities of Fig. 3 reduce to the four spin-summed quantities shown here.

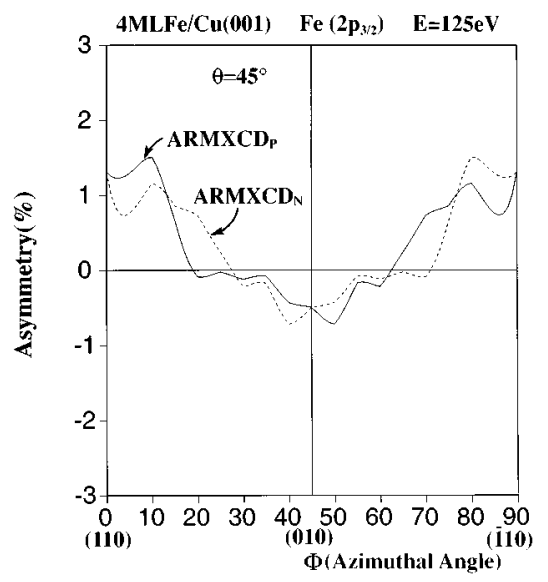

FIG. 5. Spin-summed angle-resolved magnetic circular dichroism for positive (solid line) and negative (broken line) helicities at photoemission direction $\theta=45^{\circ}$ as a function of the azimuthal direction. Note that $\mathrm{ARMXCD}_{P}$ equals $\mathrm{ARMXCD}_{N}$ along the mirror planes (110), (010), and (110).

resolved MXCD effect [similarly for the difference between the "parallel" term $I_{N}^{-}(\hat{\Omega})$ and the "antiparallel" term $\left.I_{N}^{+}(\hat{\Omega})\right]$. By contrast, the "parallel" term $I_{P}^{+}(\hat{\Omega})$ and the "antiparallel" term $I_{N}^{+}(\hat{\Omega})$ differ by the angle-resolved XCD effect [similarly for the difference between $I_{N}^{-}(\hat{\Omega})$ and $\left.I_{P}^{-}(\hat{\Omega})\right]$. We can therefore separate the two angle-resolved effects by defining the magnetic asymmetry $\mathrm{ARMXCD}_{P}$ for a positive helicity light as

$$
\operatorname{ARMXCD}_{P}(\hat{\Omega})=\frac{I_{P}^{+}(\hat{\Omega})-I_{P}^{-}(\hat{\Omega})}{I_{P}^{+}(\hat{\Omega})+I_{P}^{-}(\hat{\Omega})}
$$

Similarly, $\mathrm{ARMXCD}_{N}$ for a negative helicity light is defined as

$$
\operatorname{ARMXCD}_{N}(\hat{\Omega})=\frac{I_{N}^{-}(\hat{\Omega})-I_{N}^{+}(\hat{\Omega})}{I_{N}^{-}(\hat{\Omega})+I_{N}^{+}(\hat{\Omega})}
$$

In an experiment to study magnetic effects, one may choose to measure either $\mathrm{ARMXCD}_{P}$ or $\mathrm{ARMXCD}_{N}$. For normal incidence light on a crystal with $C_{4 v}$ symmetry, $\mathrm{ARMXCD}_{P}$ $(\theta, \varphi)=\operatorname{ARMXCD}_{N}\left(\theta, 90^{\circ}-\varphi\right)$. Here, $\varphi$ is measured from any mirror plane. In other words, one can measure $\operatorname{ARMXCD}_{P}$ in a quadrant and deduce the corresponding values of $\mathrm{ARMXCD}_{N}$. In Fig. 5, we show $\mathrm{ARMXCD}_{P}$ and $\mathrm{ARMXCD}_{N}$ at emission polar angle $\theta=45^{\circ}$ as a function of $\varphi$ for the $4 \mathrm{ML} \mathrm{Fe} / \mathrm{Cu}(001)$ system. The following properties are evident: (i) the magnetic asymmetries $\mathrm{ARMXCD}_{P, N}$ are typically $\approx 2 \%$, (ii) $\mathrm{ARMXCD}_{P}$ and $\mathrm{ARMXCD}_{N}$ are complementary in a $C_{4 v}$ system, and (iii) $\mathrm{ARMXCD}_{P}=\mathrm{ARMXCD}_{N}$ in mirror planes. In a nonmagnetic material, $\mathrm{ARMXCD}_{P}$ or $\mathrm{ARMXCD}_{N}$ is zero everywhere. The magnetic asymmetry is small because it is defined here as the fractional change in the integrated intensity from a given core level. In experiments where the asymmetry at a given electron energy is measured, the asymmetry could 


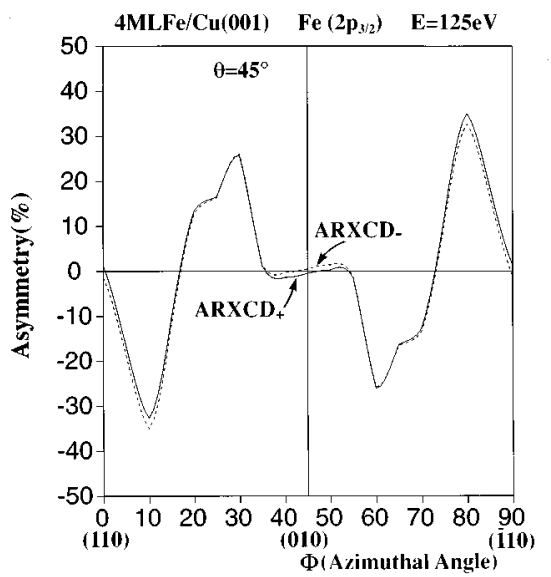

FIG. 6. Spin-summed angle-resolved circular dichroism for positive (solid line) and negative (broken line) magnetization at $\theta=45^{\circ}$ as a function of the azimuthal direction. Along the three mirror planes shown, $\mathrm{ARXCD}_{+}=-\mathrm{ARXCD}_{-}$.

be much larger (over 10\%) due to large variation in the line shape even though the asymmetry in the integrated intensity is small.

Turning now to the angle-resolved circular dichroism asymmetry, we define

$$
\operatorname{ARXCD}_{+}(\hat{\Omega})=\frac{I_{P}^{+}(\hat{\Omega})-I_{N}^{+}(\hat{\Omega})}{I_{P}^{+}(\hat{\Omega})+I_{N}^{+}(\hat{\Omega})}
$$

and

$$
\operatorname{ARXCD}_{-}(\hat{\Omega})=\frac{I_{P}^{-}(\hat{\Omega})-I_{N}^{-}(\hat{\Omega})}{I_{P}^{-}(\hat{\Omega})+I_{N}^{-}(\hat{\Omega})} .
$$

Symmetry relations for our present system with $C_{4 v}$ symmetry require that $\mathrm{ARXCD}_{+}(\theta, \varphi)=-\mathrm{ARXCD}_{-}\left(\theta, 90^{\circ}-\varphi\right)$. In Fig. 6, we show the azimuthal angular dependence of $\mathrm{ARXCD}_{+,-}$for the $4 \mathrm{ML} \mathrm{Fe} / \mathrm{Cu}(001)$ system. We note that away from symmetry directions, these quantities are as large as $\pm 35 \%$. In a nonmagnetic material, $\mathrm{ARXCD}_{+}=\mathrm{ARXCD}_{-}$ and it vanishes when measured along symmetry directions.

To make contact with quantities measured in an absorption experiment, we define an average magnetic asymmetry as

$$
\operatorname{ARMXCD}(\hat{\Omega})=1 / 2\left\{\operatorname{ARMXCD}_{P}(\hat{\Omega})+\operatorname{ARMXCD}_{N}(\hat{\Omega})\right\} .
$$

Figure 5 confirms that ARMXCD is symmetric about mirror planes. Figures 7(a), 7(b), and 7(c) show ARMXCD and its two components for general $(\theta, \varphi)$ directions in a quadrant. Similarly, we define an average angle-resolved circular dichroism asymmetry as

$$
\operatorname{ARXCD}(\hat{\Omega})=1 / 2\left\{\operatorname{ARXCD}_{+}(\hat{\Omega})+\operatorname{ARXCD}_{-}(\hat{\Omega})\right\}
$$

From Fig. 6, we see that ARXCD is antisymmetric about mirror planes. In Fig. 8, we show ARXCD for all $(\theta, \varphi)$ angles in a quadrant. Because this function is antisymmetric about mirror planes, its value is zero along such planes.
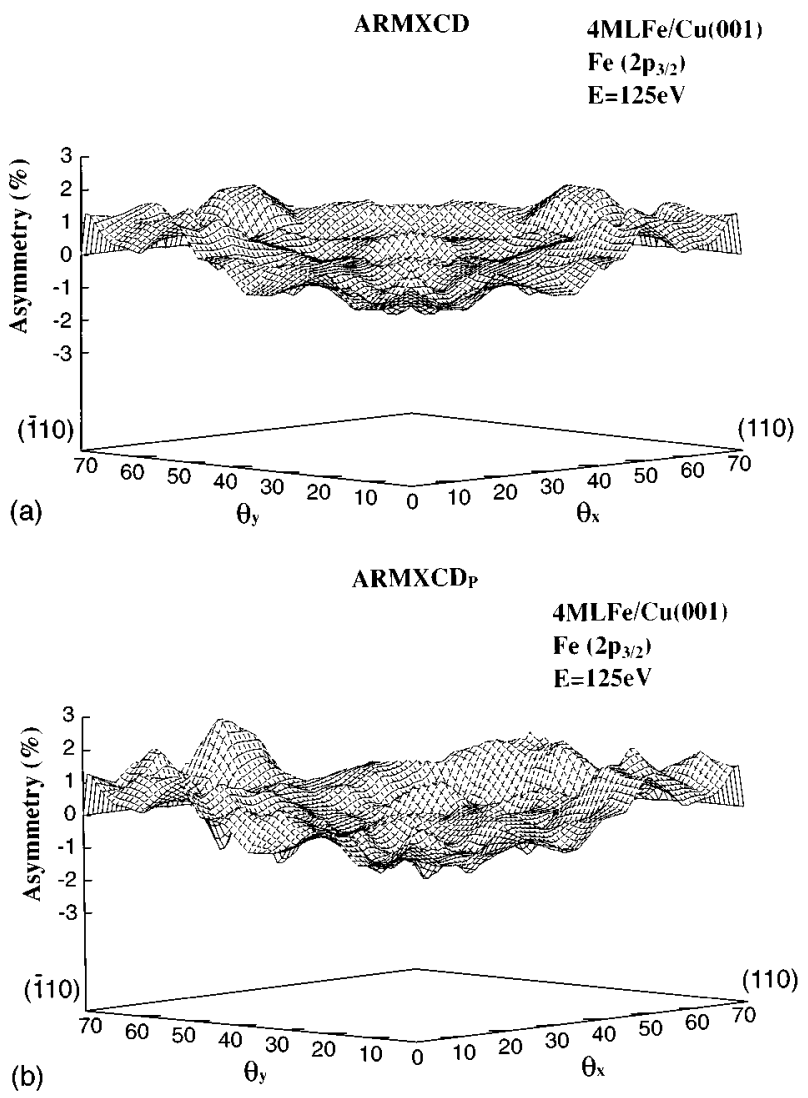

$\operatorname{ARMXCD}_{\mathrm{N}}$

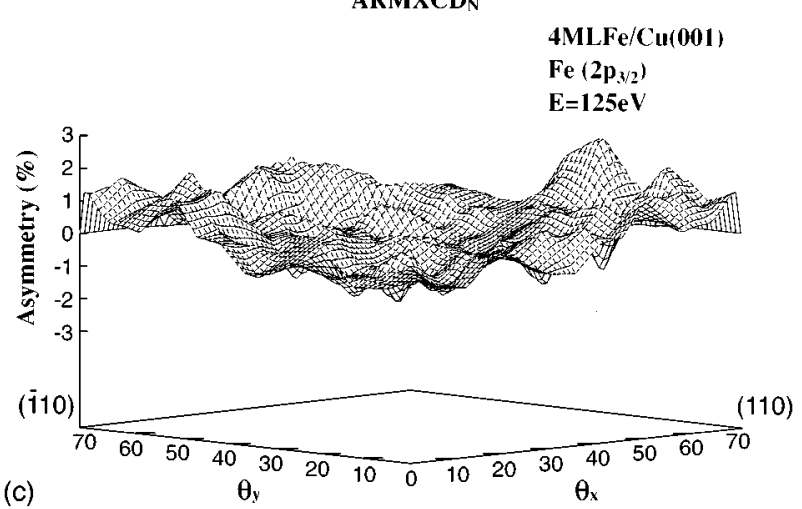

FIG. 7. Spin-summed angle-resolved magnetic circular dichroism as a function of $(\theta, \phi)$ in a quadrant bounded by the (110) and (110) azimuths, (a) for $\mathrm{ARMXCD}$, (b) for $\mathrm{ARMXCD}_{P}$, and (c) for $\operatorname{ARMXCD}_{N}$.

\section{DISCUSSION AND CONCLUDING REMARKS}

The definitions given in the last section are general and apply to arbitrary photoelectron directions. Further simplifications exist along mirror planes. From Eqs. (1)-(4), we obtain $I_{P}^{+}(\hat{\Omega})=I_{N}^{-}(\hat{\Omega})$ and $I_{P}^{-}(\hat{\Omega})=I_{N}^{+}(\hat{\Omega})$. In other words, the four curves in Fig. 4 reduce to two. Along mirror planes, we can use the parallel and antiparallel notations and define $I_{\text {parallel }}(\hat{\Omega})=I_{P}^{+}(\hat{\Omega})=I_{N}^{-}(\hat{\Omega})$, while $I_{\text {antiparallel }}(\hat{\Omega})=I_{P}^{-}(\hat{\Omega})$ $=I_{N}^{+}(\hat{\Omega})$, respectively. Using the properties already discussed in the last section, we see that along a mirror plane, $\mathrm{ARMXCD}_{P}=\mathrm{ARMXCD}_{N}=\mathrm{ARMXCD}$. Furthermore, while $\mathrm{ARXCD}_{+}$is usually much larger than $\mathrm{ARMXCD}_{P}$, however, along a mirror plane, $\mathrm{ARXCD}_{+}=\mathrm{ARMXCD}_{P}$. 


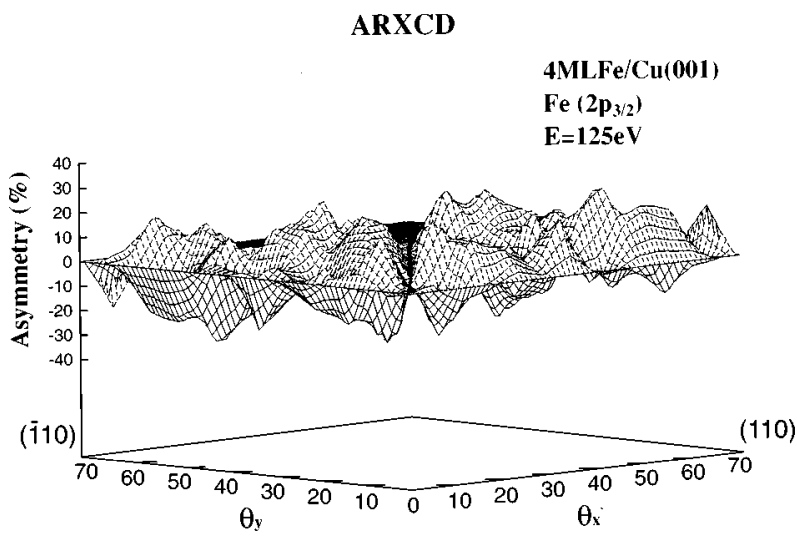

FIG. 8. Spin-summed angle-resolved circular dichroism as a function of $(\theta, \phi)$ in a quadrant bounded by the (110) and (110) azimuths.

To make contact with definitions used in an absorption experiment, the absorption magnetic x-ray circular dichroism $\sigma_{\mathrm{MXCD}}$ is simply the integral of ARMXCD over all emission angles. To see this, for a chirally invariant system, we have

$$
\int_{2 \pi} I_{P}^{+}(\hat{\Omega}) d \hat{\Omega}=\int_{2 \pi} I_{N}^{-}(\hat{\Omega}) d \hat{\Omega}=\sigma_{\text {parallel }} .
$$

Similarly, for the antiparallel quantities, we have

$$
\int_{2 \pi} I_{P}^{-}(\hat{\Omega}) d \hat{\Omega}=\int_{2 \pi} I_{N}^{+}(\hat{\Omega}) d \hat{\Omega}=\sigma_{\text {antiparallel }} .
$$

Therefore, integrating Eqs. (9) and (10) and using Eqs. (5)(8), we obtain the usual expressions of an absorption experiment:

$$
\sigma_{\mathrm{MXCD}}=\frac{\sigma_{\text {parallel }}-\sigma_{\text {antiparallel }}}{\sigma_{\text {parallel }}+\sigma_{\text {antiparallel }}}
$$

and

$$
\sigma_{\mathrm{XCD}}=0 .
$$

In practice, it is often more convenient to normalize the intensity of the $2_{p_{3 / 2}}$ state relative to that of the $2 p_{1 / 2}$ state. This was done, for example, by Waddill and co-workers. ${ }^{7-10}$ All the definitions introduced here, i.e., Eqs. (5)-(14), can be similarly normalized. For example, with normalization, Eq. (5) reads as

$$
\operatorname{ARMXCD}_{P}(\hat{\Omega})=\frac{I_{P, 3 / 2}^{+}(\hat{\Omega}) / I_{P, 1 / 2}^{+}(\hat{\Omega})-I_{P, 3 / 2}^{-}(\hat{\Omega}) / I_{P, 1 / 2}^{-}(\hat{\Omega})}{I_{P, 3 / 2}^{+}(\hat{\Omega}) / I_{P, 1 / 2}^{+}(\hat{\Omega})+I_{P, 1 / 2}^{-}(\hat{\Omega}) / I_{P, 1 / 2}^{-}(\hat{\Omega})}
$$

and Eq. (13) reads as

$$
\sigma_{\mathrm{MXCD}}=\frac{\sigma_{\text {parallel,3/2}} / \sigma_{\text {parallel, } 1 / 2}-\sigma_{\text {antiparallel,3/2 }} / \sigma_{\text {antiparallel, } 1 / 2}}{\sigma_{\text {parallel, }, 3 / 2} / \sigma_{\text {parallel, } 1 / 2}+\sigma_{\text {antiparallel, } 3 / 2} / \sigma_{\text {antiparallel }, 1 / 2}} .
$$

In conclusion, we have provided definitions for angleresolved magnetic $\mathrm{x}$-ray circular dichroism and $\mathrm{x}$-ray circular dichroism applicable to general photoelectron directions. We show that away from mirror plane directions, ARMXCD for positive or negative helicity are unequal (Fig. 5). Similarly, ARXCD for $(+)$ or $(-)$ magnetization are unequal (Fig. 6). Because of such inequalities, the definitions are necessary to specify whether an asymmetry is measured under a fixed helicity and reversing the magnetization direction; or under a fixed magnetization and reversing the helicity of the photon. The definitions are easy to use because when integrated over angles, they merge with the previously known absorption expressions. In angle-resolved asymmetry studies, surface magnetism, and surface structure can be studied as functions of the outgoing electron direction. While ARXCD, as defined here, is large along nonmirror plane directions, it does not carry magnetic information of a material. To eliminate ARXCD effects, one can make measurements confined within mirror planes. Along general directions, one can eliminate ARXCD by fixing the helicity of the light and reversing the magnetization direction, i.e., using either Eq. (5) or (6) [or the normalized form: Eq. (15)].

\section{ACKNOWLEDGMENTS}

S.Y.T. acknowledges the support of the Research Grant Council of Hong Kong. X.G. and work at the University of Wisconsin-Milwaukee are supported by the U.S. Department of Energy Contract No. DE-FG02-84ER 45076. Work by J.G.T. and G.D.W. are performed under the auspices of the U.S. Department of Energy by LLNL under Contract No. W-7405-ENG-48.

\section{APPENDIX A: RELATIVISTIC SPIN-POLARIZED PHOTOELECTRON DIFFRACTION CALCULATION}

The calculation of spin-polarized multiple-scattering photoelectron diffraction is an extension of conventional photoelectron diffraction calculation ${ }^{20,21}$ into the relativistic formalism. The relativistic Hamiltonian for an electron moving in a potential $V(\mathbf{r})$ and magnetic field $\mathbf{B}(\mathbf{r})$ can be written as $^{22-24}$

$$
H_{0}=c \boldsymbol{\alpha} \cdot \mathbf{p}+\beta m c^{2}+V(\mathbf{r})-\beta \boldsymbol{\sigma} \cdot \mathbf{B}(\mathbf{r}) .
$$

The electron-photon interaction can be regarded as a perturbation to the Hamiltonian; including up to the dipole interaction term, we have

$$
H_{I}=\boldsymbol{\sigma} \cdot \mathbf{A}
$$

where $\mathbf{A}$ is the vector potential of the incident light.

The wave function for an electron emitted via the dipole interaction is

$$
\psi\left(\mathbf{R}_{f}\right)=\int\left[\delta\left(\mathbf{R}_{f}, \mathbf{r}\right)+\int d \mathbf{r}^{\prime} G\left(\mathbf{R}_{f}, \mathbf{r}^{\prime}\right) T\left(\mathbf{r}^{\prime}, \mathbf{r}\right)\right] \psi_{D}(\mathbf{r}) d \mathbf{r}
$$

where 


$$
\begin{aligned}
\psi_{D}(\mathbf{r})= & \int\left[G_{0}\left(\mathbf{r}, \mathbf{r}^{\prime}\right)+\int G_{0}\left(\mathbf{r}, \mathbf{r}_{1}\right) t_{\mathbf{R}}\left(\mathbf{r}_{1}-\mathbf{R}, \mathbf{r}_{2}-\mathbf{R}\right)\right. \\
& \left.\times G\left(\mathbf{r}_{2}, \mathbf{r}^{\prime}\right) d \mathbf{r}_{1} d \mathbf{r}_{2}\right] H_{I} \psi_{i}\left(\mathbf{r}^{\prime}\right) d \mathbf{r}^{\prime}
\end{aligned}
$$

Here, $\psi_{i}\left(\mathbf{r}^{\prime}\right)$ is the initial state of the electron. If we expand $\psi_{D}(\mathbf{r})$ in the four-spinor relativistic form, we have

$$
\psi_{D}(\mathbf{r})=\frac{1}{r} \sum_{\kappa \mu}\left(\begin{array}{c}
f_{\kappa D}^{\mu}(r) \chi_{\kappa}^{\mu}(\hat{r}) \\
i g_{\kappa D}^{\mu}(r) \chi_{-\kappa}^{\mu}(\hat{r})
\end{array}\right),
$$

and let

$$
R_{D \kappa \mu}(r)=\frac{1}{r}\left(\begin{array}{c}
f_{\kappa D}^{\mu}(r) \\
i g_{\kappa D}^{\mu}(r)
\end{array}\right)
$$

we obtain

$$
\begin{aligned}
R_{D \kappa \mu}(r)= & \sum_{\kappa^{\prime \prime} \mu^{\prime \prime}} \int r^{\prime 2} d r^{\prime} \int\left\{G_{0 \kappa \mu}\left(r, r^{\prime}\right) \delta_{\kappa \kappa^{\prime \prime}} \delta_{\mu \mu^{\prime \prime}}\right. \\
& +\int r_{1}^{2} d r_{1} r_{2}^{2} d r_{2} G_{0 \kappa \mu}\left(r, r_{1}\right) t_{\kappa \kappa^{\prime \prime}}^{\mu \mu^{\prime \prime}}\left(r_{1}, r_{2}\right) \\
& \left.\times G_{\kappa^{\prime \prime} \mu^{\prime \prime}}\left(r_{2}, r^{\prime}\right)\right\} \psi_{E \kappa^{\prime \prime} \mu^{\prime \prime}} .
\end{aligned}
$$

Using the muffin-tin model for the scattering potential and the assumption that the magnetic field is either constant or spherically symmetric inside the muffin-tin sphere, then $\psi_{E \kappa^{\prime \prime} \mu^{\prime \prime}}$ is related to the radial part of the solution of $H_{I} \psi_{i}(\mathbf{r})$ as

$$
\psi_{E \kappa^{\prime \prime} \mu^{\prime \prime}}\left(r^{\prime}\right)=\frac{1}{r} \sum_{\kappa^{\prime} \mu^{\prime}}\left(\begin{array}{c}
f_{\kappa I}^{\mu}\left(r^{\prime}\right)\left\langle\chi_{\kappa^{\prime \prime}}^{\mu^{\prime \prime}}|\mathbf{A} \cdot \boldsymbol{\sigma}| \chi_{-\kappa^{\prime}}^{\mu^{\prime}}\right\rangle \\
i g_{\kappa I}^{\mu}\left(r^{\prime}\right)\left\langle\chi_{-\kappa^{\prime \prime}}^{\mu^{\prime \prime}}|\mathbf{A} \cdot \boldsymbol{\sigma}| \chi_{\kappa^{\prime}}^{\mu^{\prime}}\right\rangle
\end{array}\right) .
$$

Note that $\mathbf{A}$ is expressed as $A_{x}+i A_{y}$ and $A_{x}-i A_{y}$ for right and left circularly polarized light, respectively.

Using the relativistic form of the propagator $G$ and defining,

$$
\begin{aligned}
R_{F_{\kappa \kappa^{\prime}}}^{\mu \mu^{\prime} \dagger}\left(r^{\prime}\right)= & j_{\kappa \mu}^{\dagger}\left(r^{\prime}\right) \delta_{\kappa \kappa^{\prime}} \delta_{\mu \mu^{\prime}} \\
& +\int r_{1}^{2} d r_{1} r_{2}^{2} d r_{2} j_{\kappa \mu}^{\dagger}\left(r_{1}\right) t_{\kappa \kappa^{\prime}}^{\mu \mu^{\prime}}\left(r_{1}, r_{2}\right) \\
& \times G_{\kappa^{\prime} \mu^{\prime}}\left(r_{2}, r^{\prime}\right),
\end{aligned}
$$

we obtain

$$
\begin{aligned}
R_{D \kappa \mu}(r)= & \gamma h_{\kappa \mu}(r) \\
& \times\left\{\sum_{\kappa^{\prime \prime} \mu^{\prime \prime}} \int R_{F_{\kappa \kappa^{\prime}}}^{\mu \mu^{\prime} \dagger}\left(r^{\prime}\right) \psi_{E \kappa^{\prime \prime} \mu^{\prime \prime}}\left(r^{\prime}\right) r^{\prime 2} d r^{\prime}\right\} .
\end{aligned}
$$

The term in brackets is the relativistic expression for the excitation matrix, with $R_{F \kappa \kappa^{\prime}}^{\mu \mu^{\prime} \dagger}\left(r^{\prime}\right)$ reducing to $e^{i \delta L} R_{f}\left(r^{\prime}\right) \delta_{L L^{\prime}}$, in the nonrelativistic limit.

After excitation, the polarized photoelectrons are scattered inside the crystal in a manner similar to spin-polarized low-energy electron diffraction electrons. ${ }^{25}$ The single-site scattering matrix $t_{\kappa \kappa^{\prime}}^{\mu \mu^{\prime}}$ is calculated by solving the Dirac equation using a muffin-tin spin-polarized potential which is generated by self-consistent band-structure calculations. This scattering matrix is then converted to the (lms) representation and used to construct layer diffraction matrices $M_{g g^{\prime}}^{s \prime^{\prime}}$. After that, the calculation proceeds similarly to conventional photoemission, ${ }^{20,21}$ except that the matrix dimensions are doubled because spins are involved. Note that both spin-orbit and exchange effects are included because the off-diagonal elements are nonzero.

In this calculation, both the $\mathrm{Fe}$ and $\mathrm{Cu}$ potentials are obtained by the self-consistent full potential linearized augmented plane-wave band-structure method. ${ }^{26}$ The Fe potentials for spin-up and spin-down electrons are different due to exchange splitting and this difference is proportional to the magnetic moment. The direction of Fe magnetization is assumed to be perpendicular to the surface and points either outward or inward. The system has fourfold symmetry when the incident light is normal to the surface. The inner potential used is $10 \mathrm{eV}$ and inelastic damping is included via a constant imaginary potential of $4.5 \mathrm{eV}$. Up to $l=4$ is used for most calculations but $l \leqslant 6$ is used to test the convergence.
${ }^{1}$ C. T. Chen, N. V. Smith, and F. Sette, Phys. Rev. B 43, 6785 (1991)

${ }^{2}$ Y. Wu, J. Stöhr, B. D. Hermsmeier, M. G. Samant, and D. Weller, Phys. Rev. Lett. 69, 2307 (1992).

${ }^{3}$ J. G. Tobin, G. D. Waddill, and D. P. Pappas, Phys. Rev. Lett. 68, 3642 (1992).

${ }^{4}$ L. Baumgarten, C. M. Schneider, H. Petersen, F. Schäfers, and J. Kirschner, Phys. Rev. Lett. 65, 492 (1990).

${ }^{5}$ D. Venus, L. Baumgarten, C. M. Schneider, C. Boeglin, and J. Kirschner, J. Phys. C 5, 1239 (1993).

${ }^{6}$ E. Tamura, G. D. Waddill, J. G. Tobin, and P. A. Sterne, Phys. Rev. Lett. 73, 1533 (1994)

${ }^{7}$ G. D. Waddill, J. G. Tobin, X. Guo, and S. Y. Tong, Phys. Rev. B 50, 6774 (1994)

${ }^{8}$ G. D. Waddill, J. G. Tobin, X. Guo, and S. Y. Tong, J. Appl. Phys. 76, 6465 (1994).
${ }^{9}$ J. G. Tobin, E. Tamura, P. A. Sterne, G. D. Waddill, D. P. Pappas, X. Guo, and S. Y. Tong, Spectroscopy 10, 30 (1995).

${ }^{10}$ J. G. Tobin, E. Tamura, P. A. Sterne, G. D. Waddill, D. P. Pappas, X. Guo, and S. Y. Tong, J. Vac. Sci. Technol. A 13, 1534 (1995).

${ }^{11}$ G. R. Harp, S. S. P. Parkin, W. L. O'Brien, and B. P. Tonner, Phys. Rev. B 15, 3293 (1995).

${ }^{12}$ See, for example, S. Y. Tong, Surf. Sci. 299/300, 358 (1994).

${ }^{13}$ G. Schönhense, C. Westphal, J. Bansmann, M. Getzlaff, J. Noffke, and L. Fritsche, Surf. Sci. 251/252, 132 (1991).

${ }^{14}$ C. Westphal, J. Bansmann, M. Getzlaff, and G. Schönhense, Phys. Rev. Lett. 63, 151 (1989).

${ }^{15}$ J. Bansmann, M. Getzlaff, C. Westphal, F. Fegel, and G. Schönhense, Surf. Sci. 269/270, 622 (1992).

${ }^{16}$ B. T. Thole, Paolo Carra, F. Sette, and G. van der Laan, Phys. Rev. Lett. 68, 1943 (1992). 
${ }^{17}$ D. Venus, Phys. Rev. B 48, 6144 (1993).

${ }^{18}$ K. Starke, E. Navers, L. Baumgarten, and G. Kaindl, Phys. Rev. B 48, 1329 (1993).

${ }^{19}$ S. Y. Tong and H. C. Poon, Phys. Rev. B 37, 2884 (1988).

${ }^{20}$ S. Y. Tong, C. H. Li, and A. R. Lubinsky, Phys. Rev. Lett. 39, 498 (1977).

${ }^{21}$ C. H. Li, A. R. Lubinsky, and S. Y. Tong, Phys. Rev. B 17, 3128 (1978).

${ }^{22}$ R. Feder, Polarized Electrons in Surface Physics (World Scien- tific, Singapore, 1985).

${ }^{23}$ R. Feder, F. Rosicky, and B. Ackermann, Z. Phys. B 52, 31 (1983).

${ }^{24}$ B. Ackerman and R. Feder, J. Phys. C 18, 1093 (1985).

${ }^{25}$ A. Ormeci, B. H. Hall, and D. L. Mills, Phys. Rev. B 41, 4524 (1990).

${ }^{26}$ E. Wimmer, H. Krakauser, M. Weinert, and A. J. Freeman, Phys. Rev. B 24, 864 (1981); H. L. Jansen and A. J. Freeman, ibid. 30, 561 (1984). 